\title{
Axonal Protection by Nicotinamide Riboside via SIRT1-Autophagy Pathway in TNF-Induced Optic Nerve Degeneration
}

\author{
Yasushi Kitaoka $^{1}$ (D) Kana Sase $^{2} \cdot$ Chihiro Tsukahara $^{1,2} \cdot$ Naoki Fujita $^{1,2} \cdot$ Ibuki Arizono $^{1,2} \cdot$ Hitoshi Takagi $^{2}$
}

Received: 5 June 2020 / Accepted: 7 August 2020 / Published online: 20 August 2020

(C) The Author(s) 2020

\begin{abstract}
Nicotinamide adenine dinucleotide $\left(\mathrm{NAD}^{+}\right)$synthesis pathway has been involved in many biological functions. Nicotinamide riboside $(\mathrm{NR})$ is widely used as an $\mathrm{NAD}^{+}$precursor and known to increase $\mathrm{NAD}^{+}$level in several tissues. The present study aimed to examine the effect of NR on tumor necrosis factor (TNF)-induced optic nerve degeneration and to investigate whether it alters SIRT1 expression and autophagic status in optic nerve. We also examined the localization of nicotinamide riboside kinase 1 (NRK1), which is a downstream enzyme for NR biosynthesis pathway in retina and optic nerve. Intravitreal injection of TNF or TNF plus NR was performed on rats. The p62 and LC3-II protein levels were examined to evaluate autophagic flux in optic nerve. Immunohistochemical analysis was performed to localize NRK1 expression. Morphometric analysis showed substantial axonal protection by NR against TNF-induced axon loss. TNF-induced increment of p62 protein level was significantly inhibited by NR administration. NR administration alone significantly increased the LC3-II levels and reduced p62 levels compared with the basal levels, and upregulated SIRT1 levels in optic nerve. Immunohistochemical analysis showed that NRK1 exists in retinal ganglion cells (RGCs) and nerve fibers in retina and optic nerve. NR administration apparently upregulated NRK1 levels in the TNF-treated eyes as well as the control eyes. Pre-injection of an SIRT1 inhibitor resulted in a significant increase of p62 levels in the NR plus TNF treatment group, implicating that SIRT1 regulates autophagy status. In conclusion, NRK1 exists in RGCs and optic nerve axons. NR exerted protection against axon loss induced by TNF with possible involvement of upregulated NRK1 and SIRT1-autophagy pathway.
\end{abstract}

Keywords Nicotinamide riboside $\cdot$ NRK1 $\cdot$ Autophagy $\cdot$ SIRT1 $\cdot$ p62

\section{Introduction}

Nicotinamide adenine dinucleotide $\left(\mathrm{NAD}^{+}\right)$synthesis pathway has been involved in many biological functions. $\mathrm{NAD}^{+}$is synthesized by salvage of vitamin precursors, nicotinic acid (NA), nicotinamide, and nicotinamide riboside (NR). Nicotinamide phosphoribosyltransferase (Nampt) converts nicotinamide into nicotinamide mononucleotide (NMN), whereas nicotinamide riboside kinase 1 (NRK1) converts NR into NMN. Then,

Electronic supplementary material The online version of this article (https://doi.org/10.1007/s12035-020-02063-5) contains supplementary material, which is available to authorized users.

Yasushi Kitaoka

kitaoka@marianna-u.ac.jp

1 Department of Molecular Neuroscience, St. Marianna University Graduate School of Medicine, 2-16-1 Sugao, Miyamae-ku, Kaswasaki, Kanagawa 216-8511, Japan

2 Department of Ophthalmology, St. Marianna University School of Medicine, Kawasaki, Japan
NMN is converted to $\mathrm{NAD}^{+}$by nicotinamide nucleotide adenylyltransferase 1-3 (Nmnat1-3) [1]. Nmnat1-3 are enzymes which have been reported to link to axonal protection in dorsal root ganglia [2-6]. Nmnat1-3 were found to exist in optic nerve [7-9], and Nmnat2 is required to retinal ganglion cell (RGC) axon growth [9]. It was reported that cytoplasmic overexpression of Nmnat1 protected against glaucomatous RGC axon loss [10]. Our previous study demonstrated that overexpression of Nmnat3 protected against glaucomatous RGC axon loss and tumor necrosis factor (TNF)-induced axon loss [8]. On the other hand, a previous study showed a decrease in NAD level in retina in $\mathrm{DBA} / 2 \mathrm{~J}$ mice [11]. This is agreement with our previous study showing a decrease in NAD level in optic nerve in TNF-induced axon damage model [7]. Interestingly, a recent study demonstrated a significantly lower plasma nicotinamide concentration in primary open-angle glaucoma patients compared with the control group [12]. Since oral intake of vitamin B3/nicotinamide increased NAD level in retina and exerted axonal protection in $\mathrm{DBA} / 2 \mathrm{~J}$ mice [11], it is reasonable to postulate that the nicotinamide supplementation may have a beneficial effect for certain glaucomatous damages. 
$\mathrm{NR}$ is widely used as an $\mathrm{NAD}^{+}$precursor supplementation and has been reported to increase the blood $\mathrm{NAD}^{+}$level in humans [13]. NRK1 is the central ratelimiting enzyme in driving $\mathrm{NAD}^{+}$synthesis from NR [14]. However, localizations of NRK1 in retina and optic nerve have not been documented. There have been several studies suggesting critical roles of $\mathrm{NAD}^{+}$and its precursors on autophagy machinery [15-17]. Autophagy is a cellular process including the clearance of unnecessary proteins and maintains homeostasis in several types of neurons. We and others reported that autophagy plays crucial roles in certain different optic nerve damages such as optic nerve crush model, hypertensive glaucoma model, and TNF-induced axon damage model [18-21]. For autophagy research, SQSTM1/p62 is used as a marker and decrease of $\mathrm{p} 62$ level is associated with autophagy activation [22]. In yeast, NR upregulates $\mathrm{NAD}^{+}$levels, enhances Sir2 functions, and extends lifespan [23]. Sirtuin 1 (SIRT1), the mammalian homo$\log$ of yeast $\mathrm{Sir} 2$, can be activated by calorie restriction [24], $\mathrm{NAD}^{+}$, and its precursors [25]. Thus, the purpose of present study is to examine the effect of NR on TNF-induced axonal degeneration and to investigate whether it alters SIRT1 expression and autophagic status in optic nerve. We also examined the localization of NRK1, which is a downstream enzyme for NR biosynthesis pathway in retina and optic nerve as well as the alteration of NRK1 expression. Finally, we tested if an inhibitor of SIRT1 alters autophagy status.

\section{Materials and Methods}

\section{Animals}

Experiments were carried out on 8-week-old male Wistar rats. All studies were conducted according to the ARVO Statement for the Use of Animals in Ophthalmic and Vision Research and approved by Ethics Committee of the Institute of Experimental Animals of St. Marianna University School of Medicine. The animals were kept in the controlled rooms $\left(23 \pm 1{ }^{\circ} \mathrm{C}\right.$; humidity at $55 \pm 5 \%$; light from 6 a.m. to 6 p.m.).

\section{Intravitreal Administrations}

Intravitreal injection of TNF (Sigma-Aldrich, St. Lois, MO) was performed as described previously [7]. Phosphate-buffered saline (PBS) was used as a control. Anesthetization with intramuscular injections of a mixture of ketamine-xylazine was conducted. NR triflate was purchased from Toronto Research Chemicals
(North York, ON, Canada), dissolved in PBS. Concomitant injection of 2, 20, and 200 pmol of NR and $10 \mathrm{ng}$ TNF was performed intravitreally. For immunoblotting, NR alone injection was also performed. For the SIRT1 inhibitor study, EX-527 (Sigma-Aldrich) was dissolved in DMSO and 200 pmol of EX-527 or DMSO alone was injected intravitreally $10 \mathrm{~min}$ before intravitreal injection of NR plus TNF. One and 2 weeks after intravitreal injection, the rats were euthanatized with overdose of sodium pentobarbital and the eyes were enucleated.

\section{Immunoblotting}

Optic nerve specimens (4-mm lengths) were gathered and homogenized in protein extraction buffer 1 week after injection. Homogenized samples were then centrifuged at $15,000 \times \mathrm{g}$ for $15 \mathrm{~min}$ at $4{ }^{\circ} \mathrm{C}$. Protein concentrations were determined with the supernatants. Each sample $(3 \mu \mathrm{g})$ was applied and subjected to the mini gel (Bio-Rad Laboratories) and transferred to enhanced chemiluminescent membrane (EMD Millipore Corporation, Temecula, CA). The membranes were blocked with 5\% skim milk with tris buffered saline (TBS) containing Tween-20 and reacted with anti-p62 antibody (MBL Life Science, Nagoya, Japan), anti-LC3 antibody (MBL Life Science), anti-SIRT1 antibody (Santa Cruz Biotechnology), anti-NRK1 antibody (Lifespan Biosciences Inc. Seattle, WA) or anti- $\beta$-actin antibody (Sigma-Aldrich). After three times washing, the membranes were reacted with anti-rabbit or antimouse peroxidase-labeled secondary antibody (MP Biochemicals, Solo, $\mathrm{OH}$ ). Immunoblotting was visualized with a chemiluminescence detection system (ECL Plus Western Blotting Detection Reagents, Amersham Pharmacia Biotech).

\section{Immunohistochemistry}

Three eyes 1 week after intravitreal injection of NR or three normal eyes were collected and fixed by immersion in $4 \%$ paraformaldehyde, dehydrated, and embedded in paraffin. Sections were made through the optic disc and blocked with $1 \%$ bovine serum (Roche Diagnostics $\mathrm{GmbH}$, Mannheim, Germany). The primary antibodies were against NRK1 (1:100; LifeSpan BioSciences), neurofilament-L (a marker of nerve fibers; 1:100; Dako, Tokyo, Japan), or Thy-1 (a marker of RGC; 1:50; Santa Cruz Biotechnology, TX). The secondary antibodies were FITC-labeled or rhodaminelabeled antibodies (1:100; Cappel, Aurora, $\mathrm{OH})$. The sections were mounted on slides in DAPI-containing medium with cover glass. 


\section{Quantification of Optic Nerve Axons}

Optic nerve specimens (4-mm lengths from $1 \mathrm{~mm}$ behind the globe) were collected and soaked in Karnovsky's solution for $24 \mathrm{~h}$ at $4{ }^{\circ} \mathrm{C} 2$ weeks after injection. Several dehydrations were performed, and samples were embedded in acrylic resin at $70{ }^{\circ} \mathrm{C}$ two overnight. Then, samples were sectioned and stained with $1 \%$ paraphenylene-diamine (Sigma-Aldrich) in absolute methanol $[7,26]$. This can stain myelin, and five black and white images from each eye were obtained at the center and at each quadrant of the periphery with a light microscope (Olympus, Tokyo, Japan). These black and white images (each area is $5850{\mu \mathrm{m}^{2}}^{2}$, and total area is $29,250 \mu \mathrm{m}^{2}$ per eye) were used for quantification with the Aphelion image processing software (ADCIS S.A., Hérouville Saint-Clair, France). The number of axons was averaged in each eye and each group, and data were presented as the number per square millimeter. After quantification, representative color photos were obtained.

\section{Statistical Analysis}

Data are expressed as mean \pm SEM. Differences among groups were analyzed by one-way ANOVA with posthoc Tukey's HSD test or Mann-Whitney method. A probability value was considered statistically significant when $p<0.05$.
Fig. 1 Paraphenylene-diamine staining of optic nerve axons 2 weeks after injection. a Control group. b TNF-injected group. c 2 pmol NR + TNF-injected group. d 20 pmol NR + TNF-injected group. e 200 pmol NR + TNFinjected group. Scale bar $=$ $10 \mu \mathrm{m}$. (f) Morphometric analysis of axon number. (CTL: $n=5$, TNF: $n=5,2$ pmol NR + TNF: $n=6,20$ pmol NR + TNF: $n=7$, 200 pmol NR + TNF: $n=7)\left(P^{\#}\right.$ $<0.0001$ vs. CTL, $P^{*}<0.0005$ vs. TNF, $P^{* * *}<0.0001$ vs. TNF)
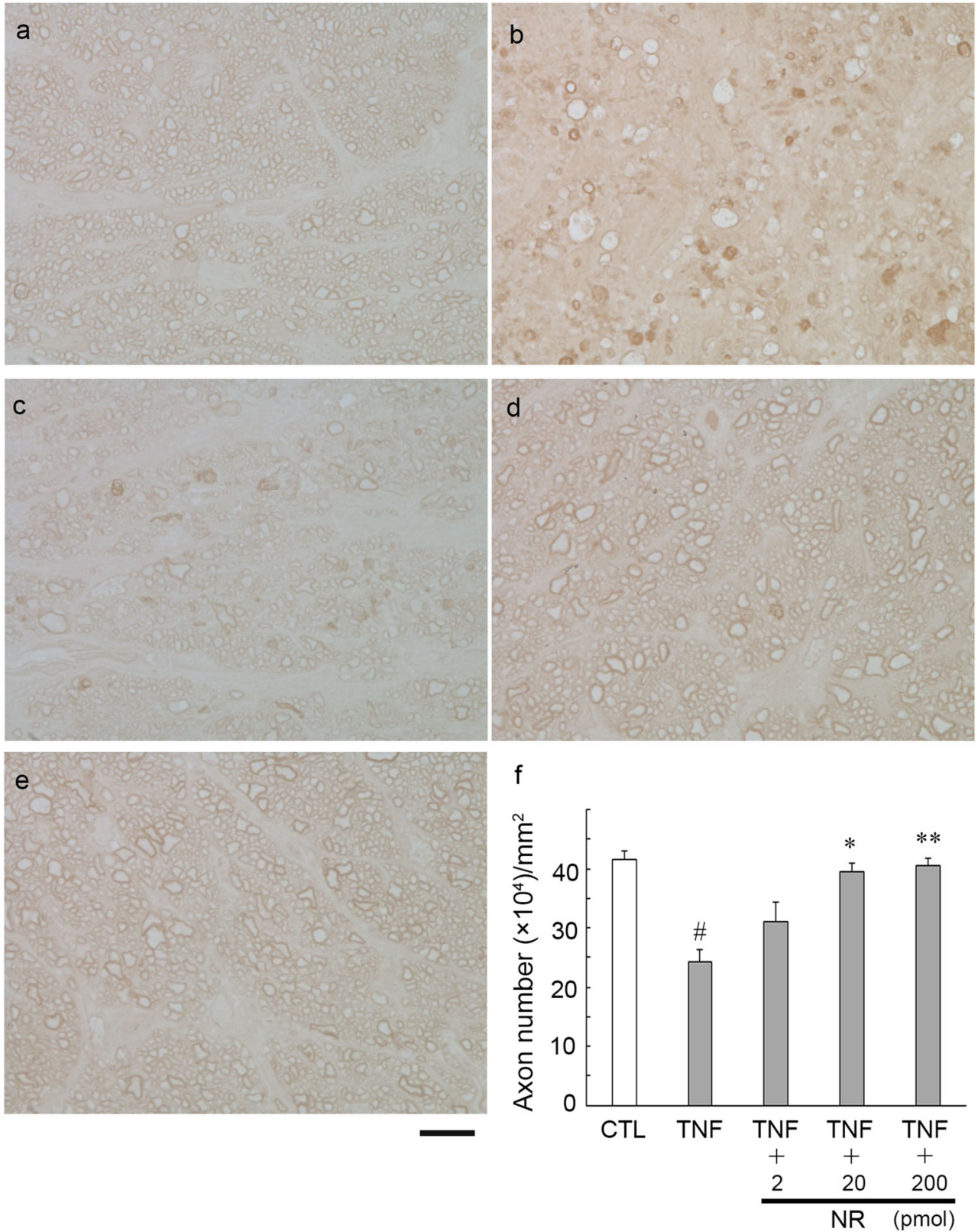


\section{Results}

\section{Effects of NR on TNF-Induced Axon Loss in Optic Nerve}

As shown previously [7], histological findings again showed substantial degenerative changes and apparent axon losses after TNF injection (Fig. 1b) compared with the control (Fig. 1a). Co-injection with 2 pmol NR plus TNF showed slightly protective tendency (Fig. 1c). However, this was not statistically significant $(p=$ 0.1850 vs. TNF; Fig. 1f). Co-injection with 20 or 200 pmol NR plus TNF showed noticeable protective effects compared with the TNF alone injection (Fig. $1 \mathrm{~d}$ and e, respectively). The quantitative analysis showed remarkable protective effects against TNFinduced axon loss, and these were statistically significant $(20$ pmol and 200 pmol NR: $p=0.0002$ vs. TNF, and $p<0.0001$ vs. TNF, respectively; Fig. 1f).

\section{Effects of TNF and NR on LC3-II Protein Levels in Optic Nerve}

There was a significant increase in the LC3-II level in the treatment with 200 pmol NR plus TNF as compared with those in the treatment with TNF at 1 week (Fig. 2a). Moreover, 200 pmol NR alone administration significantly increased the LC3-II level compared with the control group (Fig. 2b).
Effects of TNF and NR on p62 Protein Levels in Optic Nerve

In agreement with our previous findings [27], p62 protein level was significantly increased in optic nerve in TNF-treated group at 1 week (Fig. 3a). Treatment with 200 pmol NR plus TNF completely prevented this increase of p62 (Fig. 3a). In addition, 200 pmol NR alone administration significantly decreased p62 protein level compared with the control group (Fig. 3b).

\section{Effects of TNF and NR on SIRT1 Protein Levels in Optic Nerve}

As we recently found [28], no significant change in SIRT1 protein level was seen in between TNF-treated group and PBS-treated group (Fig. 4a). However, treatment with 200 pmol NR plus TNF significantly increased the SIRT1 levels compared with TNF alone treatment (Fig. 4a). Moreover, 200 pmol NR alone administration significantly upregulated the SIRT1 levels compared with the control group (Fig. 4b).

\section{NRK1 in Retina and Optic Nerve}

To examine the effect of NR and its metabolic pathway further, we investigated the localization of NRK1 in the retina and optic nerve. In the normal retina, the NRK1 immunoreactive pattern was similar to that of Thy-1 immunoreactivity (Fig. 5, upper panels). Most NRK1-positive cells were colocalized with Thy1-positive cells (Fig. 5, upper panels). The NRK1 immunoreactivity was also observed in the nerve fiber layer, and these were
Fig. 2 Immunoblotting in samples from optic nerves 1 week after injection. a Effects of TNF or 200 pmol NR + TNF on LC3-II protein level (CTL: $n=3$, TNF: $n=3, \mathrm{TNF}+\mathrm{NR}: n=3)\left(P^{* *}\right.$ $<0.005$ ). (b) Effects of $200 \mathrm{pmol}$ NR on LC3-II protein level. (CTL: $n=3, \mathrm{NR}: \mathrm{n}=3)\left(P^{*}\right.$ $<0.05$ ) a
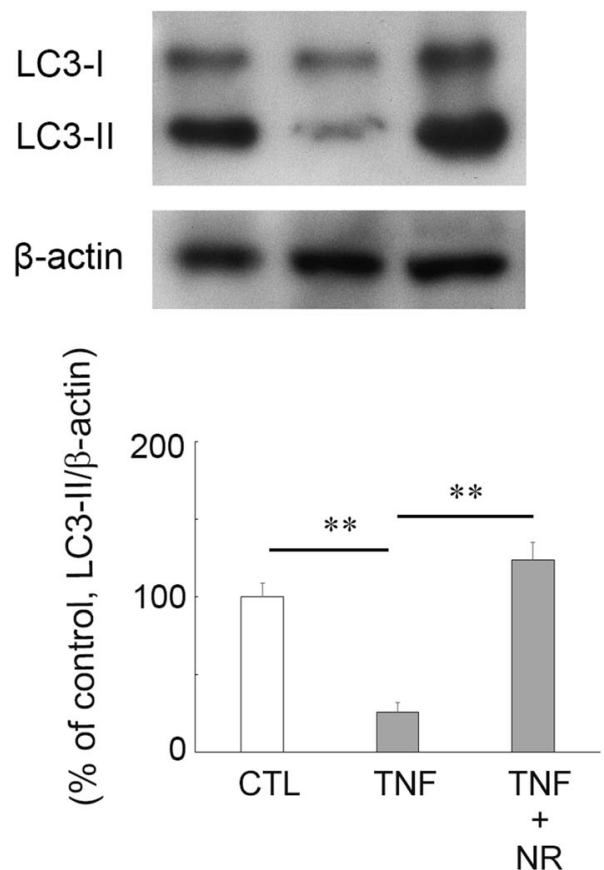
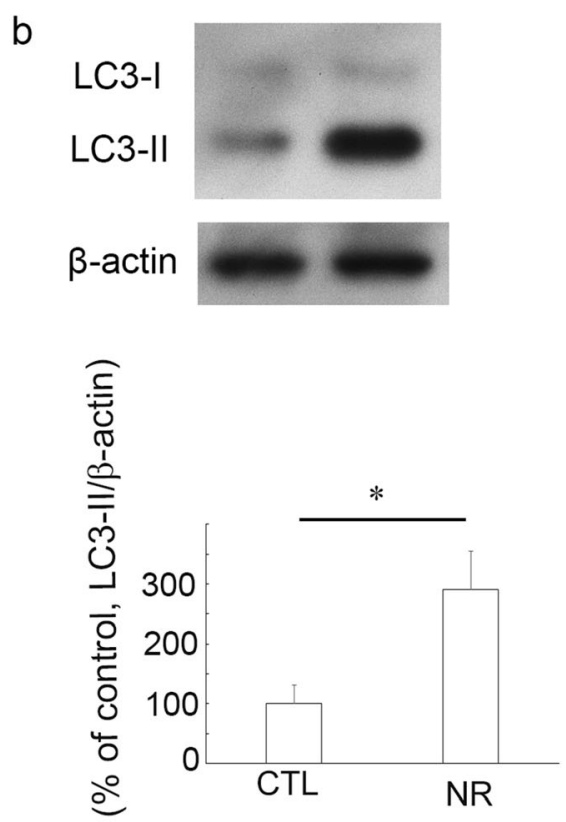
Fig. 3 Immunoblotting in samples from optic nerves 1 week after injection. a Effects of TNF or 200 pmol NR + TNF on p62 protein level. (CTL: $n=4$, TNF: $n=4$, TNF + NR: $n=4)\left(P^{* * *}\right.$ $<0.005)$. b Effects of $200 \mathrm{pmol}$ NR on p62 protein level. (CTL: $n=5$, NR: $n=5)\left(P^{*}<0.05\right)$ a

p62

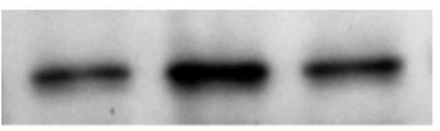

$\beta$-actin
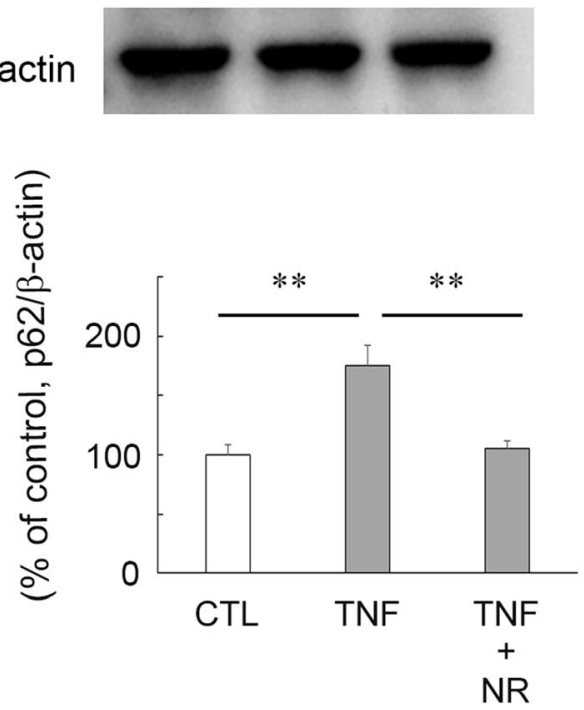

b p62

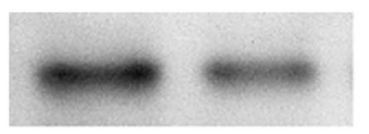

$\beta$-actin
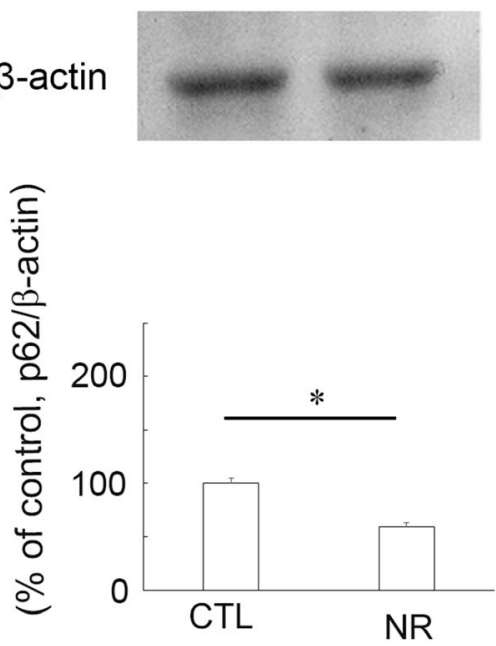

colocalized with neurofilament immunoreactivity (Fig. 5, upper middle panel). In the optic nerve, the immunoreactivity of NRK1 was modest, but some immunopositive fibers were colocalized with neurofilament immunoreactivity (Fig. 5, lower middle panel). In the optic nerve after NR treatment, a lot of NRK1 immunopositive fibers were apparently colocalized with neurofilament immunoreactivity (Fig. 5, lower panel). In the retina after NR treatment, similar findings to the normal eyes were observed (Suppl. Fig. 1).

\section{Effects of TNF and NR on NRK1 Protein Levels in Optic Nerve}

We next investigated the change in NRK1 expression in optic nerve. There was a tendency of decrease in NRK1 protein levels after TNF injection (Fig. 6a). Unexpectedly, treatment with 200 pmol NR plus TNF significantly increased the NRK1 levels compared with TNF alone treatment (Fig. 6a). Furthermore, 200 pmol NR alone administration
Fig. 4 Immunoblotting in samples from optic nerves 1 week after injection. a Effects of TNF or 200 pmol NR + TNF on SIRT1 protein level. (CTL: $n=4$, TNF: $n=4, \mathrm{TNF}+\mathrm{NR}: n=4)\left(P^{* *}\right.$ $<0.005)$. b Effects of $200 \mathrm{pmol}$ NR on SIRT1 protein level. (CTL: $n=3$, NR: $n=3)\left(P^{*}\right.$ $<0.05$ ) a

\section{SIRT1}
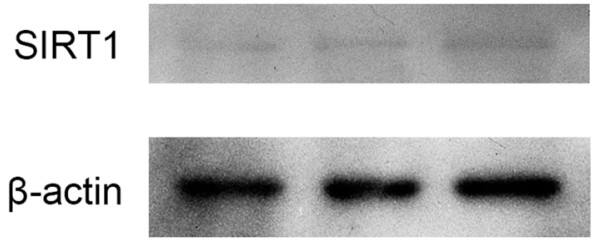

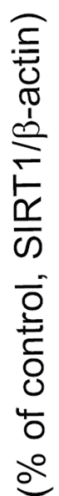

b

\section{SIRT1}
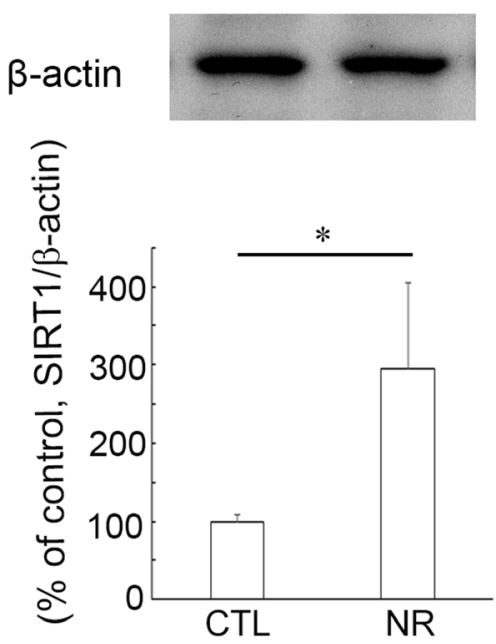
significantly upregulated the NRK1 levels compared with the control group (Fig. 6b).

\section{Effects of an SIRT1 Inhibitor on p62 Protein Level in the NR plus TNF Treatment in Optic Nerve}

To investigate whether an inhibitor of SIRT1 alters autophagy status, pre-injection of EX-527 was performed before co-injection with $200 \mathrm{pmol}$ NR and TNF. Preinjection of EX-527, an inhibitor of SIRT1, significantly upregulated p62 levels as compared with NR plus TNF treatment group (Fig. 7).

\section{Discussion}

The present study revealed that intravitreal injection of NR exerted substantial axonal protection in TNF-induced optic nerve degeneration. Recent studies have demonstrated beneficial effects of NR on central nervous system. For example, NR treatment prevented dopaminergic neuronal loss in Parkinson's disease model flies [29]. Moreover, NR treatment ameliorated selective cognitive impairment in aged mice and decreased the number of amyloid beta plaques in cortex of Alzheimer's disease model mice [30]. Furthermore, NR treatment decreased glial activation and delayed motor neuron loss
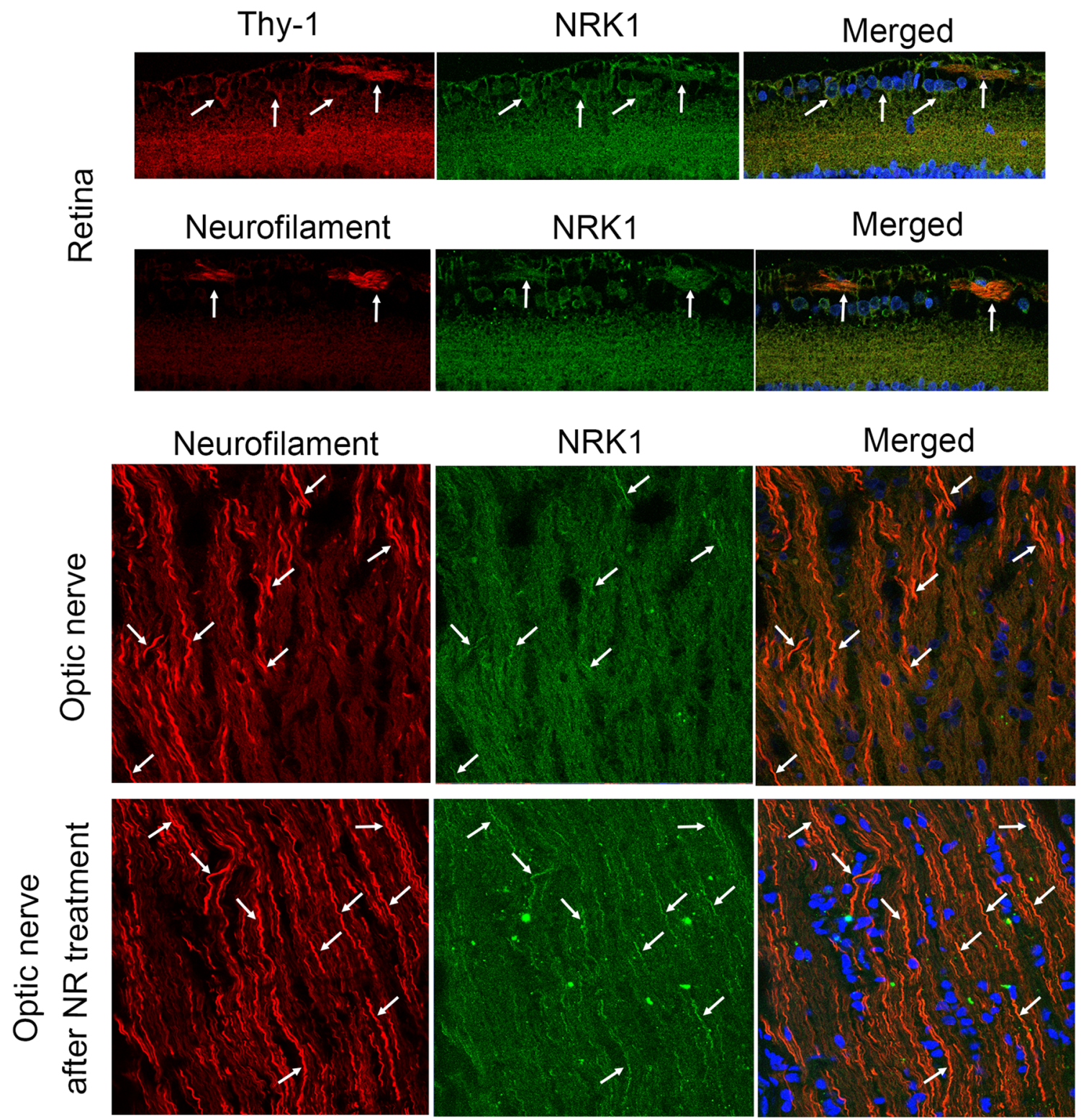

Fig. 5 Immunohistochemistry in retina and optic nerve. NRK1-positive cells were colocalized with Thy-1-positive cells in normal retina. NRK1 immunoreactivity was colocalized with neurofilament immunoreactivity in normal retina. A few NRK1 immunoreactivities were colocalized with neurofilament immunoreactivity in normal optic nerve, but a lot of NRK immunopositive fibers were colocalized with neurofilament immunoreactivity in the NR-treated optic nerve. Arrows indicate colocalization. Scale bar $=50 \mu \mathrm{m}$ 
Fig. 6 Immunoblotting in samples from optic nerves 1 week after injection. a Effects of TNF or $200 \mathrm{pmol} \mathrm{NR}+$ TNF on NRK1 protein level. (CTL: $n=4$, TNF: $n=4, \mathrm{TNF}+\mathrm{NR}: n=4)\left(P^{*}\right.$ $<0.05)$. b Effects of 200 pmol NR on NRK1 protein level. $(\mathrm{CTL}: n=3$, NR: $n=3)\left(P^{*}\right.$ $<0.05)$ a

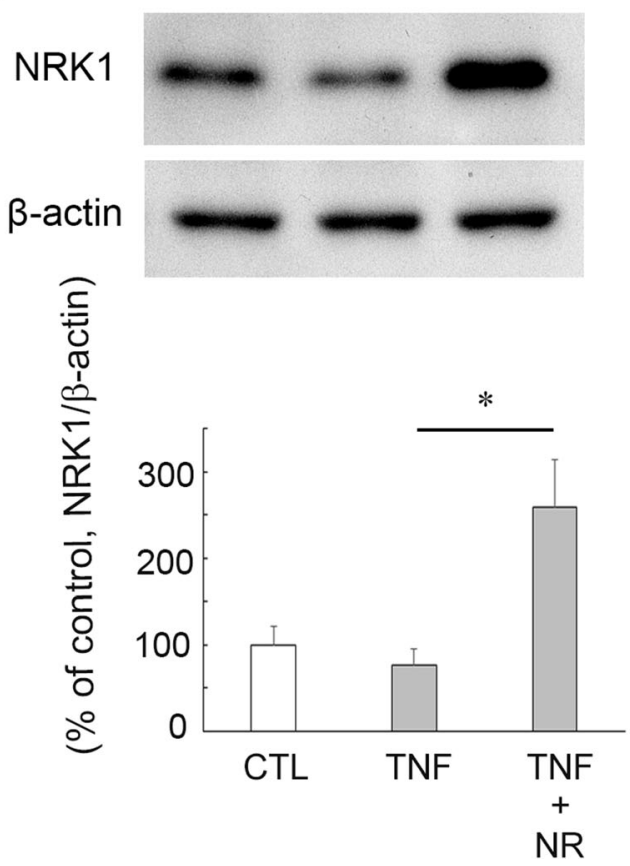

b
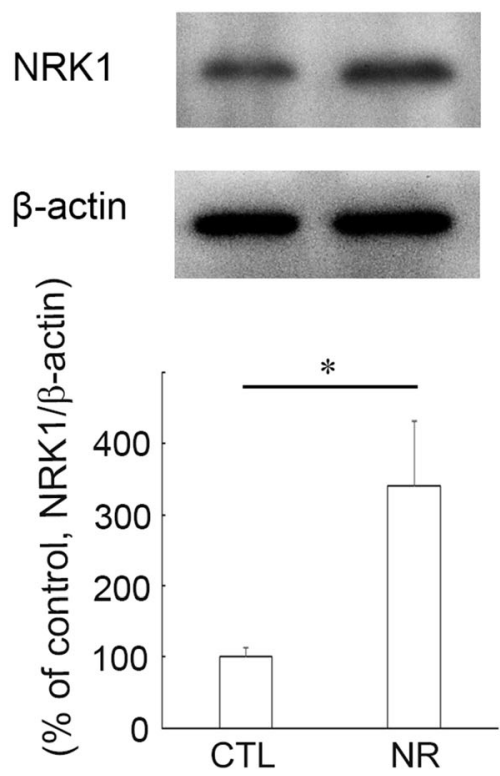

in the spinal code of amyotrophic lateral sclerosis model mice [31]. In axons, it was previously reported that NR significantly delayed axonal degeneration in dorsal root ganglia neurons [5]. More recently, it was shown that NR prevented axonal degeneration induced by excitotoxicity in cortical neurons [32]. Collectively, NR may have a protective effect on several types of axons against distinct injury models.

Our previous study suggested that upregulated p62 levels indicate impairment of autophagic flux in optic nerve $[8,18$, 27]. In the present study, upregulated p62 levels induced by TNF were significantly prevented by NR. NR significantly increased LC3-II protein levels in both the TNF-treated group and the control group. NR also significantly reduced $\mathrm{p} 62$ protein level compared with the basal level, implicating that NR can enhance autophagic flux. It is worthy to note that nicotinamide protects against palmitate-induced hepatotoxicity through SIRT1-dependent autophagy induction [33]. Since SIRT1 activators stimulate the autophagy [34, 35] and increased $\mathrm{NAD}^{+}$stimulates SIRT1 activity [36], we speculated that this pathway may exist between NR and autophagy induction. Therefore, we further examined SIRT1 expression and found that NR upregulated SIRT1 protein level in optic nerve. Consistently, a very recent study demonstrated that treatment of NR upregulated SIRT1 activity and decreased neuroinflammation in the brains of Gulf War Illness mice [37], suggesting that NR can activate SIRT1 in certain neuronal system as well as in optic nerve. Moreover, our recent study demonstrated that an SIRT1 activator exerted axonal protection with upregulated autophagic status [28]. Furthermore, the current study found that the SIRT1 inhibitor significantly upregulated p62 level in the NR plus TNF treated group, implicating that inhibition of SIRT1 leads to autophagy impairment. Taken together, these findings suggest that NR attenuated axonal degeneration via SIRT1-autophagy

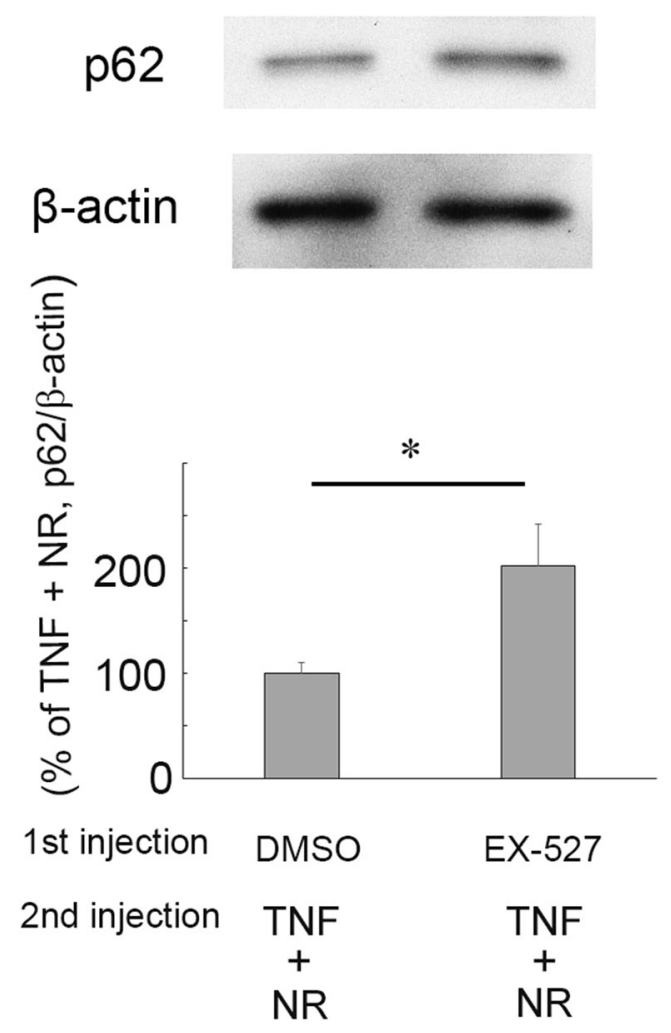

Fig. 7 Immunoblotting in samples from optic nerves 1 week after injection. Effects of EX-527 on p62 protein level in the 200 pmol NR plus TNF-treated group. (pre-injection of DMSO and TNF + NR: $n=4$, pre-injection of EX-527 and TNF + NR: $n=4)\left(P^{*}<0.05\right)$ 
pathway. In line with this concept, a previous study demonstrated the neuroprotective activity of cilostazol via SIRT1-autophagy activation in rat Parkinson's disease model [38].

Although Nmnats were found in optic nerve [7-9], NRK1 has not been examined in retina and optic nerve. Thus, the present study firstly showed that NRK1 exists in RGCs and optic nerve axons. This finding makes it possible that locally applied exogenous NR can accelerate NAD biosynthesis and activate downstream effectors. Surprisingly, NR administration clearly boosted NRK1 levels in the TNF-treated eyes as well as the control eyes. The mechanism of this regulation is unclear, while the regulation of NRK2 in muscle has been proposed in response to various conditions [39]. One hypothesis posits that exogenous NR may recruit more NRK1, thereby accelerating conversion to downstream effectors.

In conclusion, NR exerts axonal protection against TNFinduced optic nerve degeneration with the possible upregulated NRK1 and through SIRT1-autophagy pathway.

Authors' Contributions YK designed the whole study, performed the intravitreal injection, enucleated eyes, performed immunohistochemistry, and wrote the manuscript. KS performed axon counting and immunoblot analysis and interpreted data. CT performed the protein assay and prepared several samples for immunoblot and immunohistochemistry. NF performed the process for plastic sections and interpreted data. IA prepared several samples for immunoblot and immunohistochemistry. HT performed statistical analysis and wrote the manuscript. All authors have read and approved the manuscript.

Funding Information This study was supported by Grants-in-Aid from KAKENHI (18K09427 for Y.K., 17K11469 for H.T.).

Data Availability All data generated or analyzed during this study are included in this published article.

\section{Compliance with Ethical Standards}

Competing Interests The authors declare that they have no competing interests.

Ethics Approval and Consent to Participate Not applicable.

Consent for Publication Not applicable.

Open Access This article is licensed under a Creative Commons Attribution 4.0 International License, which permits use, sharing, adaptation, distribution and reproduction in any medium or format, as long as you give appropriate credit to the original author(s) and the source, provide a link to the Creative Commons licence, and indicate if changes were made. The images or other third party material in this article are included in the article's Creative Commons licence, unless indicated otherwise in a credit line to the material. If material is not included in the article's Creative Commons licence and your intended use is not permitted by statutory regulation or exceeds the permitted use, you will need to obtain permission directly from the copyright holder. To view a copy of this licence, visit http://creativecommons.org/licenses/by/4.0/.

\section{References}

1. Bogan KL, Brenner C (2008) Nicotinic acid, nicotinamide, and nicotinamide riboside: a molecular evaluation of NAD+ precursor vitamins in human nutrition. Annu Rev Nutr 28:115-130

2. Araki T, Sasaki Y, Milbrandt J (2004) Increased nuclear NAD biosynthesis and SIRT1 activation prevent axonal degeneration. Science 305:1010-1013

3. Wang J, Zhai Q, Chen Y, Lin E, Gu W, McBurney MW, He Z (2005) A local mechanism mediates NAD-dependent protection of axon degeneration. J Cell Biol 170:349-355

4. Gilley J, Coleman MP (2010) Endogenous Nmnat2 is an essential survival factor for maintenance of healthy axons. PLoS Biol 8: e1000300

5. Sasaki Y, Araki T, Milbrandt J (2006) Stimulation of nicotinamide adenine dinucleotide biosynthetic pathways delays axonal degeneration after axotomy. J Neurosci 26:8484-8491

6. Press C, Milbrandt J (2008) Nmnat delays axonal degeneration caused by mitochondrial and oxidative stress. J Neurosci 28: 4861-4871

7. Kitaoka Y, Hayashi Y, Kumai T, Takeda H, Munemasa Y, Fujino $\mathrm{H}$, Kitaoka Y, Ueno S et al (2009) Axonal and cell body protection by nicotinamide adenine dinucleotide in tumor necrosis factorinduced optic neuropathy. J Neuropathol Exp Neurol 68:915-927

8. Kitaoka Y, Munemasa Y, Kojima K, Hirano A, Ueno S, Takagi H (2013) Axonal protection by Nmnat3 overexpression with involvement of autophagy in optic nerve degeneration. Cell Death Dis 4: e860

9. Gilley J, Adalbert R, Yu G, Coleman MP (2013) Rescue of peripheral and CNS axon defects in mice lacking NMNAT2. J Neurosci 33:13410-13424

10. Zhu Y, Zhang L, Sasaki Y, Milbrandt J, Gidday JM (2013) Protection of mouse retinal ganglion cell axons and soma from glaucomatous and ischemic injury by cytoplasmic overexpression of Nmnat1. Invest Ophthalmol Vis Sci 54:25-36

11. Williams PA, Harder JM, Foxworth NE, Cochran KE, Philip VM, Porciatti V, Smithies O, John SW (2017) Vitamin B3 modulates mitochondrial vulnerability and prevents glaucoma in aged mice. Science 355:756-760

12. Kouassi Nzoughet J, Chao de la Barca JM, Guehlouz K, Leruez S, Coulbault L, Allouche S, Bocca C, Muller J et al (2019) Nicotinamide deficiency in primary open-angle glaucoma. Invest Ophthalmol Vis Sci 60:2509-2514

13. Trammell SA, Schmidt MS, Weidemann BJ, Redpath P, Jaksch F, Dellinger RW, Li Z, Abel ED et al (2016) Nicotinamide riboside is uniquely and orally bioavailable in mice and humans. Nat Commun 7:12948

14. Ratajczak J, Joffraud M, Trammell SA, Ras R, Canela N, Boutant M, Kulkarni SS, Rodrigues M et al (2016) NRK1 controls nicotinamide mononucleotide and nicotinamide riboside metabolism in mammalian cells. Nat Commun 7:13103

15. Zhu Y, Zhao KK, Tong Y, Zhou YL, Wang YX, Zhao PQ, Wang ZY (2016) Exogenous NAD(+) decreases oxidative stress and protects $\mathrm{H}_{2} \mathrm{O}_{2}$-treated RPE cells against necrotic death through the upregulation of autophagy. Sci Rep 6:26322

16. Sedlackova L, Korolchuk VI (2020) The crosstalk of NAD, ROS and autophagy in cellular health and ageing. Biogerontology 21: 381-397. https://doi.org/10.1007/s10522-020-09864-0

17. Li W, Zhu L, Ruan ZB, Wang MX, Ren Y, Lu W (2019) Nicotinamide protects chronic hypoxic myocardial cells through regulating mTOR pathway and inducing autophagy. Eur Rev Med Pharmacol Sci 23:5503-5511

18. Munemasa Y, Kitaoka Y (2015) Autophagy in axonal degeneration in glaucomatous optic neuropathy. Prog Retin Eye Res 47:1-18 
19. Koch JC, Lingor P (2016) The role of autophagy in axonal degeneration of the optic nerve. Exp Eye Res 144:81-89

20. Russo R, Nucci C, Corasaniti MT, Bagetta G, Morrone LA (2015) Autophagy dysregulation and the fate of retinal ganglion cells in glaucomatous optic neuropathy. Prog Brain Res 220:87-105

21. Adornetto A, Parisi V, Morrone LA, Corasaniti MT, Bagetta G, Tonin P, Russo R (2020) The role of autophagy in glaucomatous optic neuropathy. Front Cell Dev Biol 8:121

22. Klionsky DJ, Abdelmohsen K, Abe A, Abedin MJ, Abeliovich H, Acevedo Arozena A, Adachi H, Adams CM et al (2016) Guidelines for the use and interpretation of assays for monitoring autophagy (3rd edition). Autophagy 12:1-222

23. Belenky P, Racette FG, Bogan KL, McClure JM, Smith JS, Brenner C (2007) Nicotinamide riboside promotes Sir2 silencing and extends lifespan via Nrk and Urh1/Pnp1/Meu1 pathways to NAD+. Cell 129:473-484

24. Wolf G (2006) Calorie restriction increases life span: a molecular mechanism. Nutr Rev 64:89-92

25. Imai S, Guarente L (2014) NAD+ and Sirtuins in aging and disease. Trends Cell Biol 24:464-471

26. Sadun AA, Smith LE, Kenyon KR (1983) Paraphenylenediamine: a new method for tracing human visual pathways. J Neuropathol Exp Neurol 42:200-206

27. Sase K, Kitaoka Y, Munemasa Y, Kojima K, Takagi H (2015) Axonal protection by short-term hyperglycemia with involvement of autophagy in TNF-induced optic nerve degeneration. Front Cell Neurosci 9:425

28. Kitaoka Y, Sase K, Tsukahara C, Fujita N, Tokuda N, Kogo J, Takagi H (2020) Axonal protection by a small molecule SIRT1 activator, SRT2104, with alteration of autophagy in TNF-induced optic nerve degeneration. Jpn J Ophthalmol 64:298-303. https:// doi.org/10.1007/s10384-020-00731-6

29. Schöndorf DC, Ivanyuk D, Baden P, Sanchez-Martinez A, De Cicco S, Yu C, Giunta I, Schwarz LK et al (2018) The NAD+ precursor nicotinamide riboside rescues mitochondrial defects and neuronal loss in iPSC and fly models of Parkinson's disease. Cell Rep 23:2976-2988
30. Xie X, Gao Y, Zeng M, Wang Y, Wei TF, Lu YB, Zhang WP (2019) Nicotinamide ribose ameliorates cognitive impairment of aged and Alzheimer's disease model mice. Metab Brain Dis 34: 353-366

31. Harlan BA, Killoy KM, Pehar M, Liu L, Auwerx J, Vargas MR (2020) Evaluation of the NAD+ biosynthetic pathway in ALS patients and effect of modulating NAD+ levels in hSOD1-linked ALS mouse models. Exp Neurol 327:113219

32. Vaur P, Brugg B, Mericskay M, Li Z, Schmidt MS, Vivien D, Orset C, Jacotot E et al (2017) Nicotinamide riboside, a form of vitamin B3, protects against excitotoxicity-induced axonal degeneration. FASEB J 31:5440-5452

33. Shen C, Dou X, Ma Y, Ma W, Li S, Song Z (2017) Nicotinamide protects hepatocytes against palmitate-induced lipotoxicity via SIRT1-dependent autophagy induction. Nutr Res 40:40-47

34. Salminen A, Kaarniranta K (2009) SIRT1 regulation of longevity via autophagy. Cell Signal 21:1356-1360

35. Luo G, Jian Z, Zhu Y, Zhu Y, Chen B, Ma R, Tang F, Xiao Y (2019) Sirtl promotes autophagy and inhibits apoptosis to protect cardiomyocytes from hypoxic stress. Int J Mol Med 43:2033-2043

36. Imai S, Guarente L (2016) It takes two to tango: NAD+ and sirtuins in aging/longevity control. NPJ Aging Mech Dis 2:16017

37. Joshi U, Evans JE, Pearson A, Saltiel N, Cseresznye A, Darcey T, Ojo J, Keegan AP et al (2020) Targeting sirtuin activity with nicotinamide riboside reduces neuroinflammation in a GWI mouse model. Neurotoxicology. 79:84-94. https://doi.org/10.1016/j. neuro.2020.04.006

38. Hedya SA, Safar MM, Bahgat AK (2018) Cilostazol mediated Nurr1 and autophagy enhancement: neuroprotective activity in rat rotenone PD model. Mol Neurobiol 55:7579-7587

39. Fletcher RS, Lavery GG (2018) The emergence of the nicotinamide riboside kinases in the regulation of NAD+ metabolism. J Mol Endocrinol 61:R107-R121

Publisher's Note Springer Nature remains neutral with regard to jurisdictional claims in published maps and institutional affiliations. 